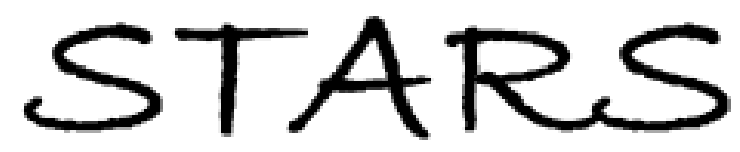

University of Central Florida

STARS

$1-1-2013$

\title{
Extension of the multiconfiguration Hartree-Fock method to allow double photoionization of atoms
}

Hari P. Saha

University of Central Florida

Find similar works at: https://stars.library.ucf.edu/facultybib2010

University of Central Florida Libraries http://library.ucf.edu

This Article is brought to you for free and open access by the Faculty Bibliography at STARS. It has been accepted for inclusion in Faculty Bibliography 2010 s by an authorized administrator of STARS. For more information, please contact STARS@ucf.edu.

\section{Recommended Citation}

Saha, Hari P., "Extension of the multiconfiguration Hartree-Fock method to allow double photoionization of atoms" (2013). Faculty Bibliography 2010s. 4632.

https://stars.library.ucf.edu/facultybib2010/4632

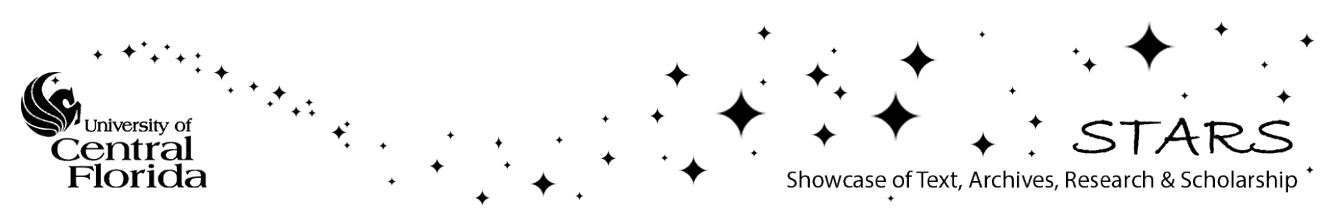




\title{
Extension of the multiconfiguration Hartree-Fock method to allow double photoionization of atoms
}

\author{
Hari P. Saha* \\ Physics Department, University of Central Florida, Orlando, Florida 32816, USA
}

(Received 2 July 2012; revised manuscript received 17 December 2012; published 12 April 2013)

\begin{abstract}
Very recently we were successful in extending the multiconfiguration Hartree-Fock (MCHF) method for electron-impact ionization of atoms to allow double photoionization of atoms. We report, as a test case, the results of our calculation of the triple differential cross section (TDCS) for double photoionization of helium. We have calculated the initial state in the Hartree-Fock and the MCHF approximations and the final state in the Coulomb approximation and the screening potential approximation in order to compare with previously published works. We compare the present results calculated at $30-\mathrm{eV}$ excess photon energy shared equally by two final-state continuum electrons, with other similar theoretical calculations. Finally, we also calculate the TDCS at $20-\mathrm{eV}$ excess photon energy to compare with experimental observation.
\end{abstract}

DOI: 10.1103/PhysRevA.87.042703

PACS number(s): $34.80 . \mathrm{Dp}$

\section{INTRODUCTION}

The double photoionization (DPI) of a helium atom has been investigated extensively both theoretically and experimentally. Briggs and Schmidt [1] gave a detailed description of the DPI process of helium and provided a discussion of different theoretical methods applied to this problem and their success in verifying the experimental measurements. Among the most accurate theoretical approaches which produce accurate results are the convergent close-coupling [2,3] and the time-dependent close-coupling (TDCC) methods [4,5]. Other theoretical methods which produce reliable results for integral cross sections are the $R$-matrix with pseudostate [6] method and the eigenchannel $R$-matrix method [7]. Some of the earlier methods which produced very good results deserve to be mentioned. Maulbetsch and Briggs [8-10] reported several results on double photoionization of helium using correlation into the final state wave function as the product of three Coulomb functions (3C) which is asymptotically correct. Teng and Shakeshaft [11], Proulx and Shakeshaft [12], and Pont and Shakeshaft [13] investigated the DPI of helium using the basis set method which included correlation through the product of two screened Coulomb functions.

Experimentally, Brauning et al. [14] measured the triple differential cross section (TDCS) for double photoionization of helium at 20-eV excess energy. Cvejanovic et al. [15] studied both experimentally and theoretically the angular correlation in the final-state two continuum electrons of the He double photoionization for equal and unequal sharing of 40-eV excess energy. Bolognesi et al. [16] studied the double photoionization of helium for unequal energy sharing of 40-eV excess photon energy by the two photoelectrons. Dawson et al. [17] measured the TDCS for double photoionization of helium at $60-\mathrm{eV}$ excess energy sharing equally and unequally by the two ejected electrons.

As already mentioned, a number of theoretical approaches have been applied to study electron correlation effects in the DPI of a helium atom. Most of them were engaged to improve the final-state wave function and study the importance of final-state electron correlation. Although few theoretical methods produce results which are in excellent agreement with

\footnotetext{
*haripada.saha@ucf.edu
}

experiment, there is not enough description of important physics which is responsible for the excellent results. Moreover, these theoretical approaches still have difficulty in explaining the experimental observations at photon energy close to threshold. In addition, the existing theoretical calculations on the heavier atom could not produce very good agreement with experimental results. These discrepancies between the experimental measurement and theoretical results inspired us to extend the MCHF method [18-21] to study the DPI of atoms. Moreover, although few ab initio methods are successful in explaining the experimental measurements, we believe that it is always good to develop a theoretical model which can explain the angular distribution of two photoelectrons very efficiently.

In this paper, we have extended the MCHF method of electron-impact ionization of atoms [18-21] to allow double photoionization of atoms. Since the primary motivation of the present calculation is to test the results obtained by the extended method, as a test case, we consider the double photoionization of a helium atom. Since calculation of TDCS is a critical test of any theory to judge its merit, we calculated the triple differential cross sections at $30-\mathrm{eV}$ excess photon energy shared equally by two final-state continuum electrons, using wave functions obtained for both the initial and the final states, each in two different approximations in order to determine the effects of electron correlation in each of the initial and the final states. The calculated TDCSs are then compared with similar results obtained by Le Rouzo and Dal Cappello [22]. As a further check, we also calculated the TDCS at 20-eV excess photon energy and $\theta_{1}=0^{\circ}$ in order to compare with experimental observation [14]. Previously, the screening potential approximation has been applied successfully by us [18-21] to describe electron-impact ionization of $\mathrm{H}$ and $\mathrm{He}$. The results were compared with other theoretical and experimental observations and were found to be in very good agreement.

\section{THEORY}

\section{A. Triple differential cross sections}

The triple differential cross section for double photoionization of atoms in a.u. is given by

$$
\frac{d^{3} \sigma}{d E_{2} d \Omega_{1} d \Omega_{2}}=4 \pi^{2} \alpha k_{1} k_{2} \omega\left|\left\langle\Psi_{f}{ }^{-}|T| \Psi_{i}{ }^{+}\right\rangle\right|^{2},
$$


where $k_{1}$ and $k_{2}$ are the momenta of the two continuum electrons in the final state. $E_{i}=\frac{k_{i}^{2}}{2}$ is the kinetic energy of the $i$ th continuum electron in the final state. $\omega$ is the photon energy, and $\alpha$ is the fine-structure constant. $T$ is the dipole operator, which for helium double photoionization is defined by

$$
T=\hat{\varepsilon} \cdot\left(\overrightarrow{r_{1}}+\overrightarrow{r_{2}}\right)
$$

in the length form and

$$
T=\frac{1}{i \omega} \hat{\varepsilon} \cdot\left(\nabla_{1}+\nabla_{2}\right)
$$

in the velocity form.

The initial-state wave function $\Psi_{i}{ }^{+}$is characterized by the orbital and spin angular momenta, $L_{0}$ and $S_{0}$, respectively. The final-state wave function $\Psi_{f}{ }^{-}$is described by the orbital and spin angular momentum $L_{c}$ and $S_{c}$ of the $(N-2)$ electrons of the core ion and by the momenta $\vec{k}_{1}$ and $\vec{k}_{2}$ and orbital angular momenta $l_{1}, l_{2}$ of the two continuum electrons. Using the partial-wave expansion of the two final-state continuum electron wave functions we expand the initial-state $\Psi_{i}{ }^{+}$and the final-state $\Psi_{f}{ }^{-}$wave functions in terms of the antisymmetrized $L-S$ coupled wave function for the $N$-electron system. The TDCS for double photoionization of helium then reduces to

$$
\sigma^{(3)}=\frac{4 \pi^{2} \alpha \omega}{\left[L_{0}\right]}\left|A\left(\hat{k_{1}} \hat{k_{2}}\right)\right|^{2},
$$

where

$$
\begin{aligned}
& A\left(\hat{k_{1}} \hat{k_{2}}\right)=\sum_{l_{1} l_{2}} \sum_{m_{1} m_{2}}(-i)^{l_{1}+l_{2}} e^{i\left(\sigma_{l_{1}}+\delta_{l_{1}}+\sigma_{l_{2}}+\delta_{l_{2}}\right)} \\
& \left(\begin{array}{llc}
l_{1} & 1 & l_{2} \\
m_{1} & 0 & -m_{2}
\end{array}\right) \\
& \times Y_{l_{1} m_{1}}\left(\theta_{1}, \phi_{1}\right)(-1)^{m_{2}} Y_{l_{2} m_{2}}^{*}\left(\theta_{2}, \phi_{2}\right)\left\langle\psi_{f}\|T\| \psi_{i}\right\rangle
\end{aligned}
$$

with $[x]=(2 x+1)$, where $\left\langle\psi_{f}\|T\| \psi_{i}\right\rangle$ is the reduced dipole matrix element.

\section{COMPUTATIONAL PROCEDURE}

Previous investigations show that both initial- and finalstate correlation are very important for double photoionization of a helium atom. Accordingly, we have the following.

\section{A. Initial state}

We first calculated the initial-state helium atom wave function in the Hartree-Fock (HF) approximation where electron correlation is neglected. We then included the electron correlation in the multiconfiguration Hartree-Fock (MCHF) approximation. It is well known that the MCHF method has been proven to be most accurate to describe the electron correlation effect in the bound state. This method calculates electron correlation completely ab initio by solving the Schrodinger equation in the form of radial coupled integrodifferential equations completely numerically and selfconsistently through the configuration interaction procedure. The beauty of the MCHF method lies in the fact that it can identify the specific configurations which are responsible for important electron correlation through the mixing coefficients of the configurations used in the MCHF expansion of the wave function. In the present case, we included the electron correlation in the MCHF approximation by expanding the helium atom wave function with excited-state configurations:

$$
\begin{aligned}
\Psi_{i}\left({ }^{1} S\right)= & c_{1} 1 s^{2}+c_{2} 2 s^{2}+C_{3} 2 p^{2}+C_{4} 3 s^{2}+c_{5} 3 p^{2} \\
& +c_{6} 3 d^{2}+c_{7} 4 s^{2}+c_{8} 4 p^{2}+c_{9} 4 d^{2}+c_{10} 4 f^{2} .
\end{aligned}
$$

The excited orbitals $2 s, 2 p, 3 s, 3 p, 3 d, 4 s, 4 p, 4 d$, and $4 f$ are calculated variationally and self-consistently completely $a b$ initio using the MCHF method. The mixing coefficients $c_{i}$ are $c_{1}=0.99596, c_{2}=-0.06177, \quad c_{3}=0.06211, c_{4}=$ $-0.00785, c_{5}=0.01105, c_{6}=-0.01275, c_{7}=-0.00171, c_{8}=$ $0.00269, c_{9}=-0.00348$, and $c_{10}=0.00416$. The binding energy of the ground state of helium is found to be -2.9029105 a.u., which is in very good agreement with the most accurate value -2.9037438 a.u. [23]. One can see that the configurations $2 s^{2}$ and $2 p^{2}$ mix reasonably well with the parent $1 s^{2}$ configuration.

\section{B. Final state}

To examine the effect of electron correlation in the final state, the final-state continuum electron wave function is calculated in two approximations-(i) the Coulomb (CLB) approximation and (ii) the screening potential (SP) approximation-in order to compare our results with those obtained with similar approximations to make sure that the extended MCHF method for double photoionization of atoms is working correctly. It should be mentioned that the screening potential approximation is an approximation to the actual Coulomb potential between the two continuum electrons. It accounts for partial electron correlation. In the screening potential approximation the exact Coulomb interaction between the two continuum electrons in the final state is replaced by a variationally determined angle dependent screening potential due to mutual screening of the nucleus by the ejected electrons using effective charges which satisfy proper asymptotic boundary conditions. On the other hand, in the Coulomb wave-function approximation we ignored this interaction between the two final-state continuum electrons. The difference between the wave functions calculated with these two approximations will determine the effect of distortion in the final-state wave functions. The screening potentials for the two continuum electrons are determined by the effective screening charges $\Delta_{1}$ and $\Delta_{2}$, which are obtained by the condition [24-27]

$$
\frac{Z_{T}-\Delta_{1}}{k_{1}}+\frac{Z_{T}-\Delta_{2}}{k_{2}}=\frac{Z_{T}}{k_{1}}+\frac{Z_{T}}{k_{2}}-\frac{1}{\vec{k}_{1}-\vec{k}_{2}},
$$

where $Z_{T}$ is the net asymptotic charge of the ionized target. The effective screening charges which satisfy the above relation are obtained as [24-27]

$$
\Delta_{i}=\frac{\left(\vec{k}_{i} \cdot \vec{k}_{i j}\right) k_{i}}{k_{i j}^{3}}(i=1,2),
$$

where $\vec{k}_{i j}=\vec{k}_{i}-\vec{k}_{j}, j \neq i$, with $k_{i j}=\left|\vec{k}_{i j}\right|$. 
The wave functions for each of the final-state continuum electrons in the SP approximation are calculated using the same numerical procedure as adopted in the multiconfiguration Hartree-Fock method [21,28] for bound and continuum electrons at each relative angle between the two continuum electrons ejected at equal energy $E_{1}=E_{2}=15 \mathrm{eV}$ for the angular momentum $l=0-6$ for the partial-wave $L S={ }^{1} P$. The description of the method was reported earlier [28].

It should be mentioned that from the physical point of view the final-state wave function accounting for electron correlation should depend on the relative angle of emission $\theta_{12}$ between the two continuum electrons. The dipole matrix elements are calculated using the correlated initial- and finalstate wave functions. Since the dipole matrix element is also angle dependent, it provides better physical understanding of the electron correlation for the TDCS as a function of the relative angle $\theta_{12}$ compared to that calculated with the angle independent dipole matrix element.

\section{RESULTS AND DISCUSSION}

As stated earlier, as a test case, we calculated triple differential cross sections in both length and velocity forms for $30-\mathrm{eV}$ excess photon energy sharing equally by the two final-state continuum electrons. The corresponding photon energy is $109 \mathrm{eV}$ where the experimental integral cross section is almost maximum [29]. Unfortunately there exist no experimental results for the TDCS for double photoionization of helium at this photon energy.

In Figs. 1-3 we have presented the polar plot of the present triple differential cross section in the velocity form for double photoionization of helium. To determine the effect of electron correlation we presented the results of the TDCS with the initial state calculated in the MCHF approximations and with the final state in the SP approximation. Considering the direction of $\hat{\varepsilon}$ as the polar axis and fixing the direction of $k_{1}$ at $0^{\circ}, 45^{\circ}$, and $90^{\circ}$, respectively, the TDCSs are

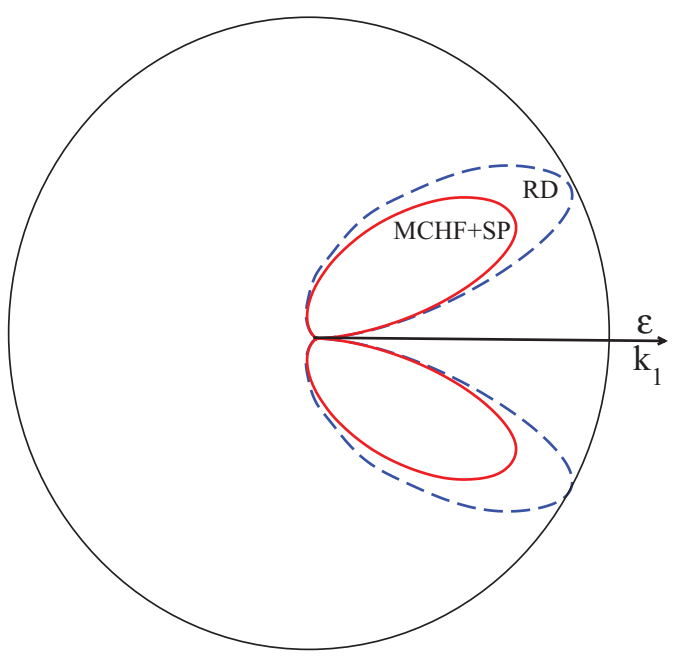

FIG. 1. (Color online) Comparison of the velocity form of $\mathrm{MCHF}+\mathrm{SP}$ TDCS results at 30-eV excess photon energy with those obtained by Le Rouzo and Dal Cappello [22] for the direction of $\hat{k}_{1}$ fixed at $0^{\circ}$ presented as a function of the direction of $\hat{k_{2}}$ of the other electron.

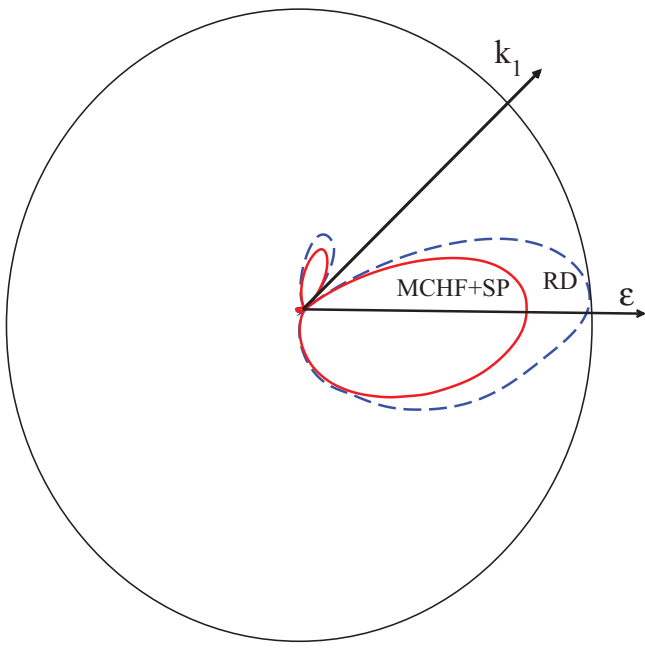

FIG. 2. (Color online) Same as Fig. 1, but for the direction of $\hat{k_{1}}$ fixed at $45^{\circ}$.

plotted as a function of the direction of $k_{2}$. Our results are compared with those obtained by Le Rouzo and Dal Cappello [22], who used the multiconfiguration self-consistent field (MCSCF) approximation for the initial state and product of two Coulomb functions with effective charges as the final state. We have found that our results compare very well. The primary difference between the two results is due to the fact that present results are obtained with continuum orbitals orthogonalized with $1 s, 2 s, 2 p, 3 s, 3 p, 3 d$, and $4 f$ orbitals of a $\mathrm{He}^{+}$ion whereas Le Rouzo and Dal Cappello results are obtained with continuum orbitals orthogonalized with the $1 s$ orbital of a He $\mathrm{He}^{+}$ion. Also, Le Rouzo and Dal Cappello used a different set of configurations in the initial state and angular momentum for the final-state continuum electrons. Moreover, the numerical procedure is quite different, since in the present calculation the wave functions in the initial and final states are calculated, respectively, in the MCHF and the SP approximations completely numerically self-consistently $a b$ initio. Overall the agreement is very good.

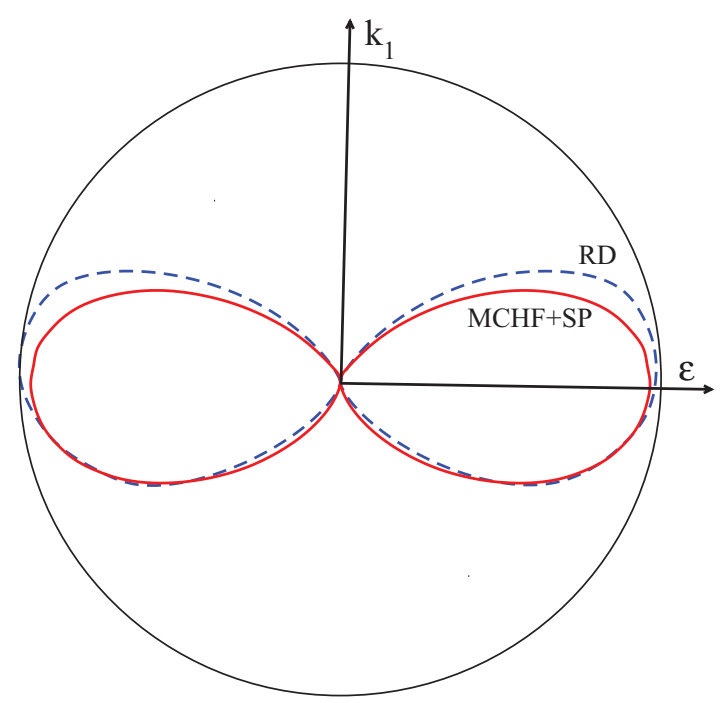

FIG. 3. (Color online) Same as Fig. 1, but for the direction of $\hat{k_{1}}$ fixed at $90^{\circ}$. 


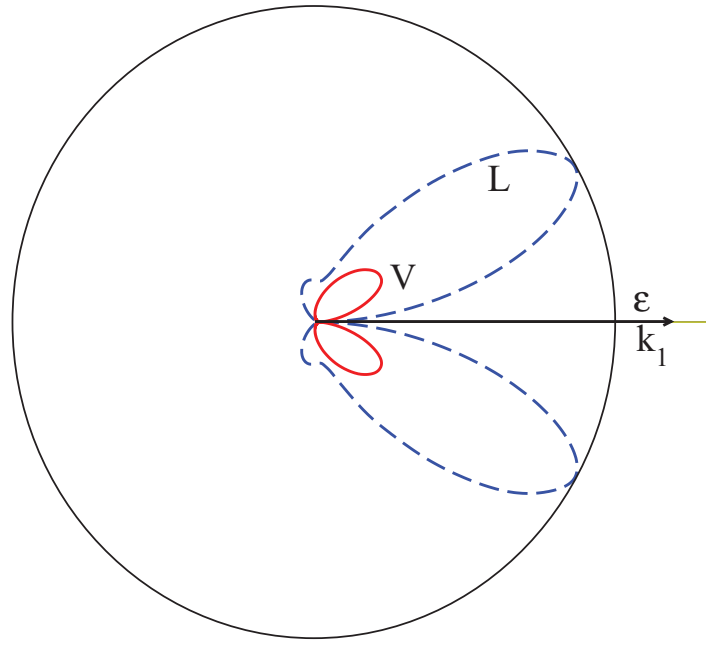

FIG. 4. (Color online) Comparison of length and velocity forms of MCHF + SP TDCS results at 30-eV excess photon energy for the direction of $\hat{k_{1}}$ fixed at $0^{\circ}$ presented as a function of the direction of $\hat{k_{2}}$ of the other electron.

In Figs. 4-6 the comparisons between the length and the velocity forms of MCHF + SP results are, respectively, presented for three directions of $k_{1}$. It is seen that length results are much larger than the velocity contribution. It should be noted that the length and velocity forms of the cross sections will be equal only when the initial- and final-state wave functions are exact solutions of the same Hamiltonian. Although the initial-state wave function calculated using the MCHF approximation is very accurate, the final-state wave function calculated using the angle dependent screening potential (SP) is an approximate wave function. It accounts for electron correlation between the two continuum electrons only partially. Accordingly, the length and velocity forms of the cross section did not give identical results. Moreover, at $109-\mathrm{eV}$ photon energy the experimental integral cross section is maximum [29], which partly explains the large difference between the length and the velocity results of the TDCS in the

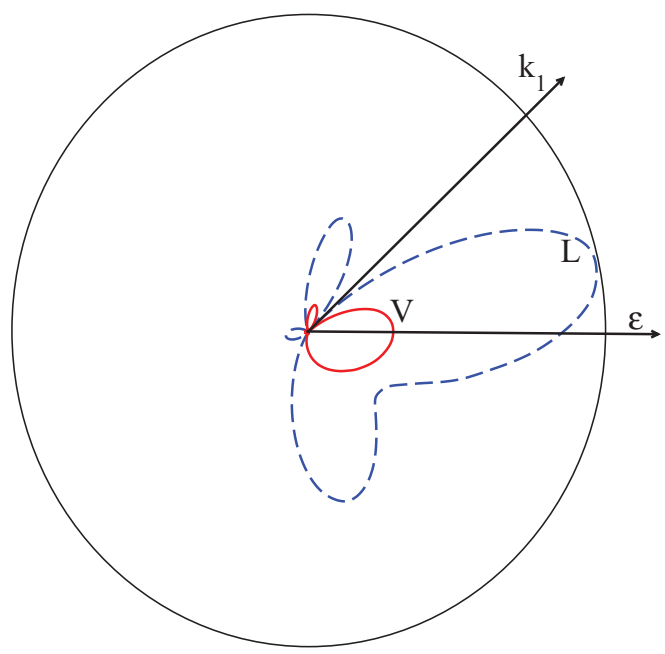

FIG. 5. (Color online) Same as Fig. 4, but for the direction of $\hat{k_{1}}$ fixed at $45^{\circ}$.

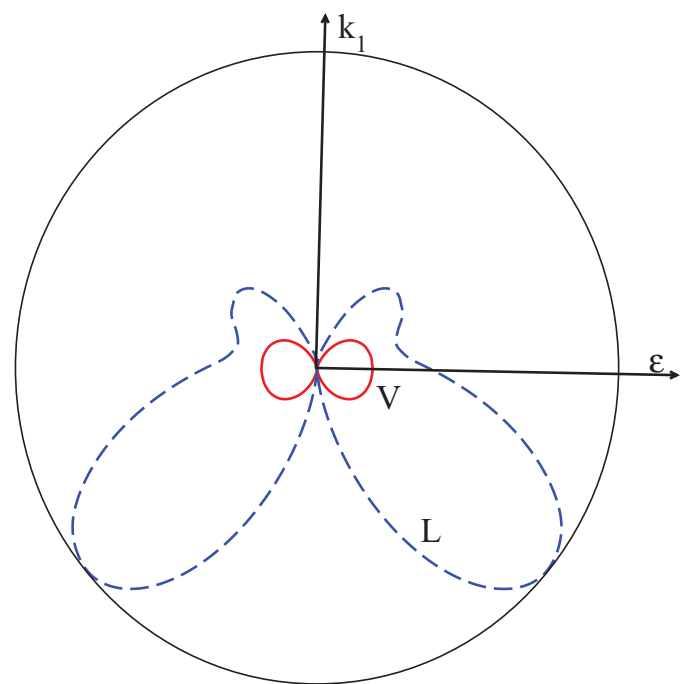

FIG. 6. (Color online) Same as Fig. 4, but for the direction of $\hat{k_{1}}$ fixed at $90^{\circ}$.

present case. The difference would be smaller at lower and at higher photon energies.

In addition, in the length form the contribution to the dipole matrix element comes from large values of $r$, whereas the contribution to the velocity form of the matrix element comes from the intermediate values of $r$. As stated by Kheifets and Bray [30] and mentioned by Bethe and Salpeter [31], the ground-state wave function calculated variationally is inaccurate at large distances. It is accurate in the intermediate distances. Accordingly, since TDCS in the present case is calculated with the initial state computed using the variational MCHF approximation, the length form of the TDCS whose contribution comes from the large distances may not be as reliable as the velocity form of the TDCS whose contribution comes from the wave function from the intermediate distances. This partly explains the disagreement between the present length and velocity results.

It should be mentioned that to describe the double photoionization of atoms Maulbetsch and Briggs [9,32] used the product of three Coulomb functions (3C) as the final-state wave functions, which is correct at large distances but not correct near the origin and intermediate distances. According to Kheifets and Bray [2] the length and velocity forms of the TDCS calculated by Maulbetsch and Briggs $[9,32]$ in the 3C approximation vary in magnitude by approximately a factor of 10. Pont and Shakeshaft [33] used the product of two screened Coulomb functions and calculated the TDCS in the velocity form only. Colgan and Pindzola [5] used the length gauge to study double photoionization of atoms. According to them their results are gauge invariant. Marchalant and Bartschat [6] used the $R$-matrix with pseudostate method to describe double photoionization of helium and found that length results are much larger than the velocity contribution. Accordingly they consider velocity calculation to be more reliable.

In Figs. 7-9 we have presented the polar plot of the TDCS in the velocity form for double photoionization of helium with the initial state calculated in the HF and the MCHF approximations and the final state in the screening potential approximation as a function of the direction of $k_{2}$ of one of the final-state electrons 


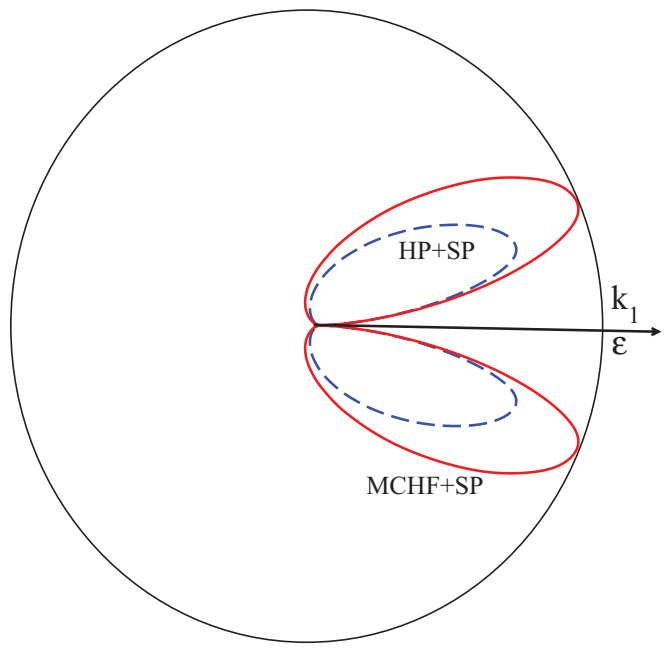

FIG. 7. (Color online) Comparison of $\mathrm{HP}+\mathrm{SP}$ and $\mathrm{MCHF}+\mathrm{SP}$ results for the direction of $\hat{k_{1}}$ fixed at $0^{\circ}$ showing the effects of electron correlation in the initial state.

in order to determine the effects of electron correlation in the initial state. The direction of $k_{1}$ of the other electron is fixed respectively at $0^{\circ}, 45^{\circ}$, and $90^{\circ}$ with respect to the polar axis in the direction of $\hat{\varepsilon}$. Comparison between the HF $+\mathrm{SP}$ and $\mathrm{MCHF}+\mathrm{SP}$ results presented in Figs. 7-9 shows the effect of electron correlation in the initial state. One can notice from the figures that the effect of electron correlation in the TDCS due to the initial state is considerably large. This is due to the fact that the configurations $2 s^{2}$ and $2 p^{2}$ in the MCHF expansion of the initial-state wave function mix reasonably well with the parent configuration. Moreover, with the increase of configurations in the initial state, more matrix elements contribute into the TDCS, resulting in increase in the TDCS approximately 1.4 times.

Figures 10-12 represent the polar plot of the triple differential cross section in the velocity form for $\theta_{1}=0^{\circ}, 45^{\circ}$, and $90^{\circ}$, respectively, for double photoionization of helium. To determine the effect of electron correlation in the final state we presented the results of the TDCS calculated with the initial

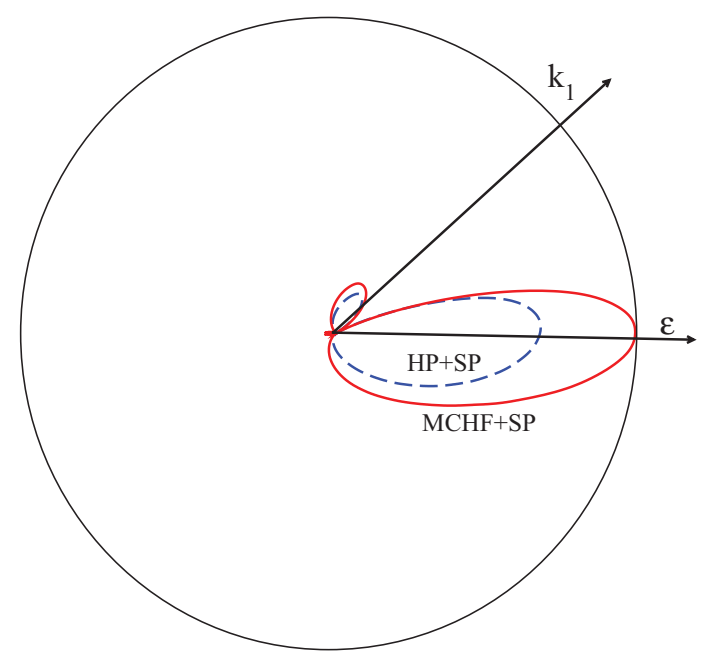

FIG. 8. (Color online) Same as Fig. 7, but for the direction of $\hat{k_{1}}$ fixed at $45^{\circ}$.

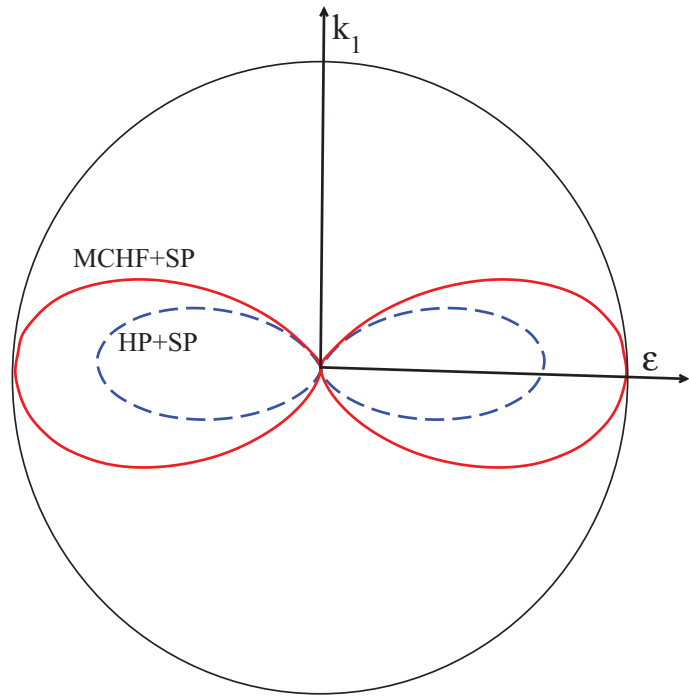

FIG. 9. (Color online) Same as Fig. 7, but for the direction of $\hat{k_{1}}$ fixed at $90^{\circ}$.

state in the MCHF approximation and the final state as the product of two Coulomb functions (CLB) as well as in the SP approximation. We would like to point out that the results of the TDCS reported by Le Rouzo and Dal Cappello [22] as well as the present results obtained with the final state calculated with the product of two Coulomb functions is incorrect, as when one electron is ejected along the direction of polarization the other electron is also ejected along the same direction of polarization with the same energy, as shown in Fig. 10. This indicates that the effect of electron correlation in the final state must be taken into account in order to obtain correct results for the TDCS for double photoionization of helium. One must notice that inclusion of electron correlation in the final state using SP approximation represents physically correct results, showing that both electrons are not ejected along the same direction of polarization with the same energy, unlike the TDCS calculated without electron correlation in the final state.

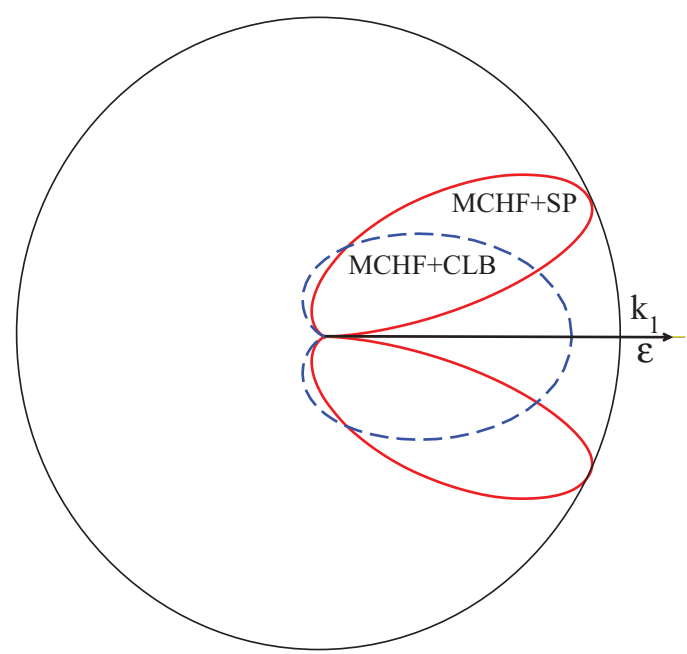

FIG. 10. (Color online) Comparison of MCHF + CLB and $\mathrm{MCHF}+\mathrm{SP}$ results, for the direction of $\hat{k_{1}}$ fixed at $0^{\circ}$ showing the effects of electron correlation in the final state. 


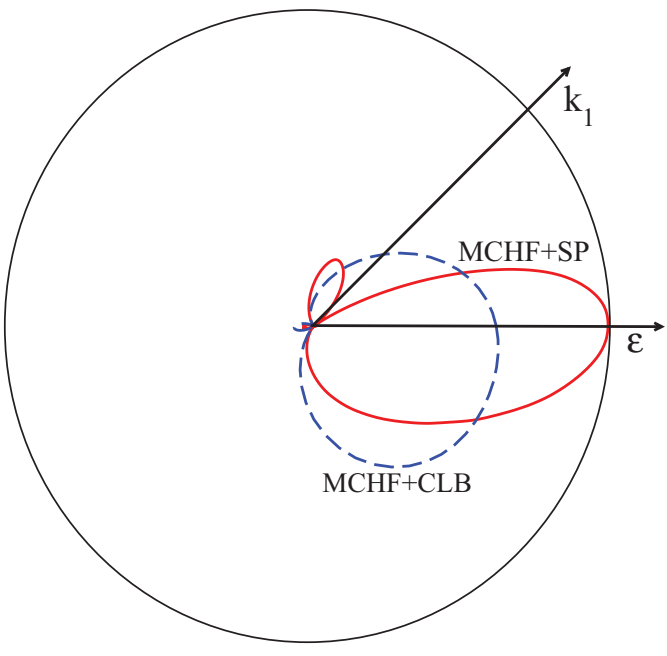

FIG. 11. (Color online) Same as Fig. 10, for the direction of $\hat{k_{1}}$ fixed at $45^{\circ}$.

One can also notice from the figures that there is a large difference between the MCHF + CLB and the MCHF + SP results. This is obviously due to the electron correlation effects due to the final state. The results without the final-state correlation differ both in shape and size. As mentioned earlier, in the SP approximation for the final state we used the angle dependent screening potential, which definitely explains better physics than averaging the potential over the angles between the two final-state continuum electrons. In fact, the electron correlation between the two final-state electrons depends on the relative angle of ejection between them. One can notice from Fig. 10 that the TDCS is minimum close to $\theta_{12}=180^{\circ}$, which explains that when the two electrons are ejected in the opposite direction $\theta_{12}=180^{\circ}$ the electron correlation between the two electrons should be minimum; on the other hand, when the two electrons are leaving, making smaller angles, the electron correlation effects should be larger. This can be seen from the figures.

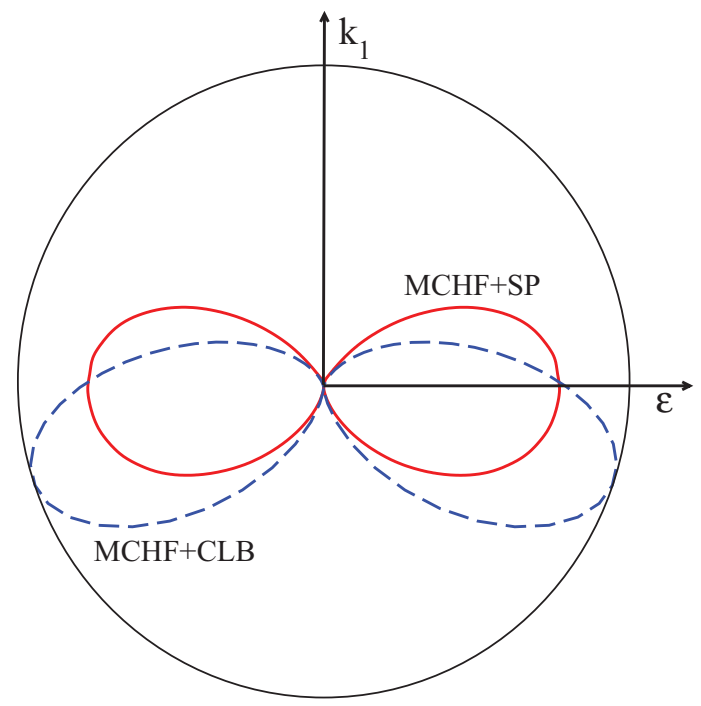

FIG. 12. (Color online) Same as Fig. 10, but for the direction of $\hat{k_{1}}$ fixed at $90^{\circ}$.

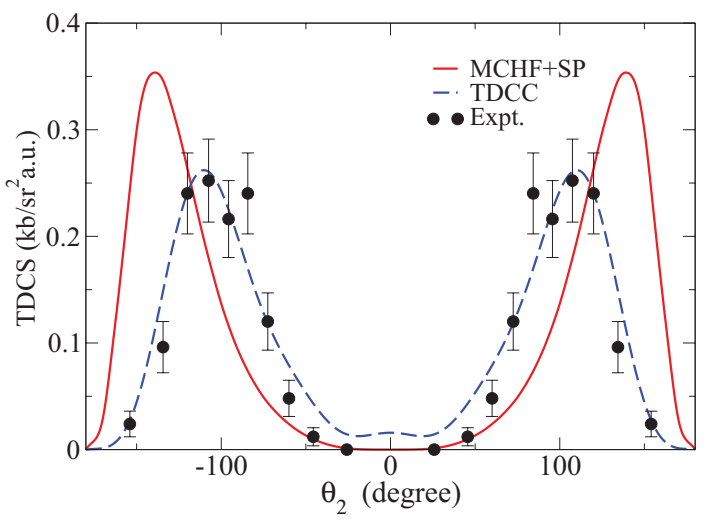

FIG. 13. (Color online) Comparison of MCHF + SP TDCS results at 20-eV excess photon energy with expt. [14] and those obtained by Colgan et al. [4] for the direction of $\hat{k_{1}}$ fixed at $0^{\circ}$.

To determine the accuracy of the approximations used for the initial and the final states we also performed a similar calculation at 20-eV excess photon energy, for which experimental results are available. Figure 13 shows the comparison of triple differential cross sections calculated at $20-\mathrm{eV}$ excess photon energy sharing equally by the two final-state continuum electrons, with experimental results of Brauning et al. [14]. In the figure, also shown are the theoretical results obtained by Colgan et al. [4], who used time-dependent close-coupling (TDCC) approximation. One can notice that there is a qualitative agreement between present results and the experiment. It is also seen from the figure that the experimental maximum occurs around $\theta_{2}= \pm 107.5^{\circ}$ whereas present results show the maximum around $\theta_{2}= \pm 140^{\circ}$.

Although it is found that the present TDCS results for double photoionization of helium agree very well with the results obtained by Le Rouzo and Dal Cappello [22] and also agree qualitatively with experiment, in order to check the present results further and make sure that the present results agree with the available calculations we integrated the TDCS results numerically over the solid angles $d \Omega_{1}$ and $d \Omega_{2}$ for $E_{1}=E_{2}=15$ and $10 \mathrm{eV}$ to obtain the singly differential cross section. In Table I we compared the singly differential cross section calculated in the velocity $(V)$ form in the equal energy case $E_{1}=\frac{E}{2}$ for double photoionization at both 30 - and $20-\mathrm{eV}$ excess energies with the results obtained in the velocity form by Pont and Shakeshaft [34] at $E_{1}=15 \mathrm{eV}$ and by Colgan et al. [4] at $10 \mathrm{eV}$, respectively. We notice that the present velocity contributions calculated in the MCHF $+\mathrm{SP}$ approximation at both excess energies $E=30$ and $20 \mathrm{eV}$ agree very well with the one obtained by Pont and Shakeshaft [34] and by Colgan et al. [4].

TABLE I. Comparison of singly differential cross sections (Mb/a.u.) in the equal-energy sharing case $E_{1}=\frac{E}{2}$ for double photoionization of helium.

\begin{tabular}{llr}
\hline \hline Excess energy $(\mathrm{eV})$ & MCHF + SP & Other theory \\
\hline 30 & $0.01653(V)$ & $0.01504(V)[34]$ \\
20 & $0.02010(V)$ & $0.0210[4]$ \\
\hline \hline
\end{tabular}




\section{CONCLUSION}

In this paper we have successfully extended the MCHF method for electron-impact ionization of atoms [18-21] to calculate the double photoionization of atoms. As a test case, we have applied the method to calculate double photoionization of a helium atom at 109-eV photon energy and found that the present TDCS results agree very well with those obtained by Le Rouzo and Dal Cappello [22], who used similar approximations. Also, the agreement between present single differential cross sections and those obtained by Pont and Shakeshaft [34] at $E_{1}=15 \mathrm{eV}$ and by Colgan et al. [4] at $E_{1}=10 \mathrm{eV}$ is very good.

Since the MCHF method for a bound-state wave function has the reputation of taking into account the electron correlation very accurately by solving the coupled radial integrodifferential equations for the bound orbitals numerically and self-consistently completely $a b$ initio through the configuration interaction procedure, we believe that the wave function and energy of the initial state are very accurate. The calculation also identifies the configurations which are responsible for electron correlation in the initial state. The final-state electron correlation is accounted for with the variationally determined angle dependent screening potential. The wave function calculated using this potential is a function of the relative angle of ejection between the two outgoing electrons. This clearly demonstrates that the electron correlation should depend on the relative angle of emission and provides a more correct physical explanation than the angle independent wave functions.

Although our extended MCHF method produces results of the TDCS calculated at 30-eV excess energy, which agree very well with those obtained by Le Rouzo and Dal Cappello [22], and also at 20-eV excess energy, which agree qualitatively with experiment, we think that further investigation is necessary in order to judge the merit of the SP approximation, which accounts for electron correlation in the final state.

We have seen that in the case of single photoionization of atoms, the MCHF method [35] for bound and continuum wave functions has the potential of taking into account the electron correlation in the initial state and electron correlation and polarization in the final state completely $a b$ initio by optimizing both bound and continuum electron orbitals simultaneously and self-consistently at each kinetic energy of the continuum electron. In the near future, we plan to include electron correlation in the initial state using the MCHF approximations and between the two final-state electrons by adding excited bound-state configurations in the expansion of the final-state continuum wave function [28] and study the effects of electron correlation and polarization to the triple differential, single differential, and total cross sections for double photoionization of different targets including heavier atoms for a number of photon energies where experimental results are available. We would like to emphasize that the extended MCHF method is more general. It will not be limited to only the DPI of helium; it can be applied to the DPI of any atom. We hope that the present investigation provides valuable information about the application of the SP approximation to account for electron correlation in the final state for triple differential cross sections in the case of double photoionization of helium atoms.

\section{ACKNOWLEDGMENTS}

We thank Dr. J. Colgan for providing us with the calculated data. We express our gratitude to Professor Robin Shakeshaft for useful discussions.
[1] J. S. Briggs and V. Schmidt, J. Phys. B 33, R1 (2000).

[2] A. S. Kheifets and I. Bray, J. Phys. B 31, L447 (1998).

[3] A. S. Kheifets and I. Bray, Phys. Rev. A 57, 2590 (1998).

[4] J. Colgan, M. S. Pindzola, and F. Robicheaux, J. Phys. B 34, L457 (2001).

[5] J. Colgan and M. S. Pindzola, Phys. Rev. A 65, 022709 (2002).

[6] P. J. Marchalant and K. Bartschat, Phys. Rev. A 56, 1697 (1997).

[7] K. W. Meyer and C. H. Greene, Phys. Rev. A 50, R3573 (1994).

[8] F. Maulbetsch and J. S. Briggs, Phys. Rev. Lett. 68, 2004 (1992).

[9] F. Maulbetsch and J. S. Briggs, J. Phys. B 26, L647 (1993).

[10] F. Maulbetsch and J. S. Briggs, J. Phys. B 27, 4095 (1994).

[11] Z.-J. Teng and R. Shakeshaft, Phys. Rev. A 47, R3487 (1993).

[12] D. Proulx and R. Shakeshaft, Phys. Rev. A 48, R875 (1993).

[13] M. Pont and R. Shakeshaft, Phys. Rev. A 51, R2676 (1995).

[14] H. Brauning et al., J. Phys. B 31, 5149 (1998).

[15] S. Cvejanovic et al., J. Phys. B 33, 265 (2000).

[16] P. Bolognesi et al., J. Phys. B 34, 3193 (2001).

[17] C. Dawson et al., J. Phys. B 34, L525 (2001).

[18] H. P. Saha, J. Phys. B 41, 055201 (2008).

[19] H. P. Saha, Phys. Rev. A 77, 062705 (2008).

[20] H. P. Saha, Phys. Rev. A 82, 042703 (2010).
[21] H. P. Saha, J. Phys. B 44, 065202 (2011).

[22] H. Le Rouzo and C. Dal Cappello, Phys. Rev. A 43, 318 (1991).

[23] K. Frankowski and C. L. Pekeris, Phys. Rev. 146, 46 (1966).

[24] C. Pan and A. F. Starace, Phys. Rev. Lett. 67, 185 (1991).

[25] C. Pan and A. F. Starace, Phys. Rev. A 45, 4588 (1992).

[26] S. Jetzke, J. Zeremba, and F. H. M. Faisal, Z. Phys. D 11, 63 (1989)

[27] F. H. M Faisal, in Atoms in Strong Fields, edited by C. A. Nicolaides, C. W. Clark, and M. H. Nayfeh (Plenum, New York, 1990), pp. 407-424.

[28] H. P. Saha and D. J. Murray, J. Phys. B 38, 3015 (2005).

[29] J. A. R. Samson, W. C. Stolte, Z. X. He, J. N. Cutler, Y. Lu, and R. J. Bartlett, Phys. Rev. A 57, 1906 (1998).

[30] A. S. Kheifets and I. Bray, Phys. Rev. A 54, R995 (1996).

[31] H. A. Bethe and E. E. Salpeter, Quantum Mechanics of One and Two Electron Atoms (Springer-Verlag, New York, 1957).

[32] F. Maulbetsch and J. S. Briggs, J. Phys. B 26, 1679 (1993).

[33] M. Pont and R. Shakeshaft, Phys. Rev. A 2, 1448 (1996).

[34] M. Pont and R. Shakeshaft, J. Phys. B 28, L571 (1995).

[35] H. P. Saha, Phys. Rev. A 66, 010702 (2002). 